\title{
Philosophiques
}

\section{Brezis, David, Kierkegaard et les figures de la paternité, Paris, Cerf, La « nuit surveillée ", 1999, 389 pages.}

\section{Dominic Desroches}

Volume 29, numéro 1, printemps 2002

Spinoza sous le prisme de son anthropologie

URI : https://id.erudit.org/iderudit/009576ar

DOI : https://doi.org/10.7202/009576ar

Aller au sommaire du numéro

Éditeur(s)

Société de philosophie du Québec

ISSN

0316-2923 (imprimé)

1492-1391 (numérique)

Découvrir la revue

Citer ce compte rendu

Desroches, D. (2002). Compte rendu de [Brezis, David, Kierkegaard et les figures de la paternité, Paris, Cerf, La « nuit surveillée », 1999, 389 pages.]

Philosophiques, 29(1), 163-165. https://doi.org/10.7202/009576ar d'utilisation que vous pouvez consulter en ligne.

https://apropos.erudit.org/fr/usagers/politique-dutilisation/ 
passages cités, des noms anciens et un troisième de noms modernes de la philosophie et de l'érudition. La seule lacune technique à signaler est l'absence d'une bibliographie des textes utilisés.

Rappelons que la mise en plein jour du problème de la prééminence de l'ontologie ou de l'hénologie l'une sur l'autre remonte à la parution, en Allemagne, du livre d'Albert Zimmermann, Ontologie oder Metaphysik (E.J. Brill, Leiden-Köln, 1965) qui ouvrait la discussion amorcée aux XIII ${ }^{\mathrm{e}}$ et $\mathrm{XIV}^{\mathrm{e}}$ siècles sur l'objet véritable de la métaphysique. Il fut suivi de près par celui de Hans Joachim Krämer, Der Ursprung der Geistmetaphysik (B.R. Grüner, Amsterdam, 1967). Nous renvoyons, pour la clarification de l'Ereignis chez Heidegger, à l'article de Philippe Verstraeten paru dans Les Études philosophiques ( ${ }^{\circ}$ 66, janvier-mars 1986, p. 113-133) : "Le sens de l'Ereignis dans Temps et être ".

DANIEL MAZILU

Université de Montréal

\section{Brezis, David, Kierkegaard et les figures de la paternité, Paris, Cerf, La « nuit surveillée », 1999, 389 pages.}

Chargé de recherche au CNRS, David Brezis nous offre ici un travail d'une grande qualité. Dans la foulée de ses travaux précédents (Temps et présence. Essai sur la conceptualité kierkegaardienne, Paris, Vrin 1989), Brezis poursuit son étude de Kierkegaard en réinterprétant les catégories philosophiques à la lumière tamisée de la biographie de Kierkegaard. Le projet de l'A. est énoncé au début de l'ouvrage : "Réinscrivant l'œuvre kierkegaardienne dans les strictes limites de son espace textuel, nous cherchons moins à en dégager l'unité fondamentale qu'à la relire à partir de tout ce qui la met fondamentalement en contradiction avec elle-même. La soumettant à une interprétation strictement immanente, nous proposons un Kierkegaard par lui-même qui est aussi, à chaque instant, un Kierkegaard contre lui-même " (p. 10). Il s'agira d'éviter l'erreur de lire Kierkegaard en "pur » philosophe ou en "pur » biographe : on voudra plutôt analyser sa pensée dans son rapport aux figures paternelles à travers leurs " traces textuelles ", c'est-à-dire de mettre son existence en correspondance avec sa conceptualité.

L'A. explorera la pensée de Kierkegaard en plaçant au centre de son étude la figure du père, figure décisive de l'œuvre s'il en est une, tout en cherchant à " mettre en question " le logos unique assurant l'unité de tout le discours philosophique (p. 15). Il le fera en débordant la figure du père vers d'autres figures auxiliaires, comme celle du frère de Søren, Peter, l'évêque Mynster, Hegel et Dieu. Cela dit, voyons plus exactement comment l'ouvrage est construit et de quelle manière il entend cerner la pensée de Kierkegaard dans ses rapports multiples et équivoques au père.

Les deux premiers chapitres traitent de points biographiques bien connus de l'œuvre, à savoir le "Tremblement de terre » et le drame des fiançailles (pp. 17-104). On y apprend que Kierkegaard fait très tôt une découverte qui influencera sa pensée : la défaillance du père. Cette découverte de la faiblesse de l'autorité, que Kierkegaard interprète comme une tromperie, se mesure à la manière dont il se positionne face à d'autres figures de l'autorité : l'évêque Mynster, Hegel et Dieu. La conséquence est décisive pour l'œuvre : le père, comme Mynster, prêchent d'agir en conformité avec la 
Parole, mais ils ne le font pas eux-mêmes. Il y a tromperie partout. Peut-être cette "infidélité » (Svigefuldhed) explique-t-elle les fiançailles rompues de Kierkegaard? Face à la tromperie, Kierkegaard optera, d'après l'A., pour le "secret de l'intériorité ».

Le chapitre III s'engage dans la voie du refus de la paternité par Kierkegaard (pp. 105-137). L'A. y explique de quelle manière Kierkegaard, qui éprouve le "fardeau paternel ", refuse de reproduire avec Régine le rapport qu'il a eu avec l'autorité trompeuse, c'est-à-dire avec son père et l'évêque Mynster. Si Kierkegaard a reconnu la « trop forte » impression du père — il lui doit sa mélancolie (Tungsind) et qu'il a su très tôt être voué au sacrifice, en revanche, selon l'A., cela ne fait que fragiliser son lien avec Régine : il n'a pas la spontanéité et la légèreté pour la rendre heureuse. Kierkegaard ne peut donc se marier, victime qu'il est du lourd et ruineux héritage paternel (pp. 128-129). Cette épreuve, insoutenable et difficile, sera vécue à travers une " contemporanéité ", notion capitale de la pensée kierkegaardienne, pour laquelle la mort du Christ reste un événement significatif pour toutes les générations.

Les chapitres IV et V s'attachent à dépister les souffrances de Kierkegaard émanant des rapports conflictuels avec le père (pp. 145-223). À travers un vaste réseau de correspondances, ils montrent que si le mal est héréditaire chez Kierkegaard (le fils prodigue), ce dernier ne peut s'empêcher de le partager avec le père. Ici encore, l'analyse interne du corpus permet de mieux comprendre des événements à première vue banals de l'existence de Kierkegaard et qui orientent pourtant sa conceptualité, par exemple la question du mariage, la rupture, le deuil et les rapports à l'Église.

Le chapitre suivant se penche sur la puissance du père comme séducteur (pp. 223-252). L'A. y situe le rapport maître-disciple illustré par les figures " séductrices " de Socrate, de Constantin dans la Répétition et de Johannes dans le " Journal du séducteur ». Le projet de Brezis implique qu'au chapitre VIII il aborde les "doubles ", puisque la figure paternelle ne saurait s'entendre de manière univoque (pp. 263-320). C'est là que l'on réalise toute l'importance du paradoxe projeté par la figure du père : tantôt il est affligé et mélancolique, tantôt il est jouisseur et mondain, ce qui ne peut qu'envoyer une image contradictoire au fils. Si l'A. se demande quel est le « vrai » visage du père, il préfère cependant connecter l'ambivalence paternelle au principe luthérien de l' "intériorité cachée » défendu par Kierkegaard pour souligner ensuite que la pseudonymie relève de ce rapport "dédoublé », lequel aura des conséquences manifestes dans la pensée religieuse de Kierkegaard et son rapport à Dieu.

L'enquête menée avec une intuition remarquable par Brezis nous mène enfin, en toute cohérence d'ailleurs, aux deux fils du père Michäel Petersen, Peter et Søren (pp. 327-383). Le plus jeune, Søren, se reconnaît comme " favori du père » et fut marqué par Peter, qu'il veut voir comme aimable (Kjerlige) ou cordial (Hjertelige), alors qu'il se réserve le qualificatif de prodige. L'A. tente de saisir comment le père composa avec ses fils et de quelle façon Søren dépend des liens unissant le frère et le père. Il conclut en rappelant que si la figure paternelle joue un rôle de premier plan dans la pensée de Kierkegaard, celle-ci se rapproche alors d'autres pensées majeures de la philosophie occidentale, comme celle de Platon, Kant, Nietzsche, Heidegger... (p. 387).

Le mérite de Brezis est de rattacher la conceptualité kierkegaardienne à sa racine vécue, existentielle, plus exactement psychologique, en retraçant les conflits générateurs de l'œuvre du philosophe danois. Brezis, qui retraduit lui-même les passages délicats pour plus de précisions, connaît à fond Kierkegaard et s'avère un guide averti dans l'interprétation des jeux de miroir et des messages contradictoires. Il parvient ainsi à donner un sens à plusieurs événements marquants de la vie de Søren Kier- 
kegaard, tout en jetant un éclairage neuf sur certaines notions oubliées, comme le renferment sur soi, le don et la dette infinie. On doit reconnaître que l'A. gagne son pari, c'est-à-dire qu' " il montre comment la biographie de Kierkegaard se prête, au plan même de la chronologie, à une analyse structurale » (p. 325).

Après la très longue lecture de l'ouvrage, nous nous demanderons toutefois si l'enquête psychologique ne met pas trop l'accent sur le bios au point d'oublier le logos. Le fait de suivre une thématique, aussi riche soit-elle, nous empêche de bien saisir, contrairement à ce qu'affirme l'A., la chronologie des événements de la vie de Kierkegaard. En utilisant les Papirer comme source première, nous avons parfois l'impression que la philosophie de Kierkegaard, que cherche à saisir l'A., disparaît dans la grille qu'il plaque sur l'œuvre. On regrettera que l'analyse ne prenne pas suffisamment en compte les pseudonymes, qui sont autant de visages que d'auteurs, autant de noms que de positions dans la topologie existentielle. Mais cela ne saurait voiler l'intérêt de l'ouvrage qui, dans les études kierkegaardiennes, aura une place de choix. Il faudrait même remercier l'A. d'avoir confronté si intelligemment la pensée de Kierkegaard à elle-même, de l'avoir mesurée à des passages rares et pertinents, de l'avoir inscrite dans sa possibilité existentielle, ce qui rappelle que la philosophie de Kierkegaard est non seulement organisée méthodiquement et structurellement, mais qu'elle est d'une complexité irréductible qu'il faut plus que jamais chercher à penser.

DOMINIC DESROCHES

Université de Montréal

\section{Bergen, Véronique, L'ontologie de Gilles Deleuze, Paris, L'Harmattan, 2001, 793 pages.}

La question de l'ontologie deleuzienne est sur le point de devenir un thème classique de la philosophie contemporaine continentale. On sait que la pensée de Deleuze s'inscrit dans le mouvement des philosophies dites «post-structuralistes " dont la méthode consiste à détruire l'idéal identitaire et la conscience historique en vue de saisir la nature événementielle du devenir compris comme suite de répétitions non identiques. Deleuze, à l'instar de quelques-uns de ses contemporains (Derrida, Lyotard, Foucault), est un philosophe de la différence unilatérale pour qui le plus déterminant pour la pensée demeure toujours lié à des rencontres inconditionnées. Dans ce contexte où règne en maître la discontinuité historique, subsiste-t-il une relation univoque entre les événements ? Quelle est la nature des enchaînements événementiels ? En quoi consiste le temps de l'événement ? Comment articuler l'être et la notion d'événement ? Voilà quelques-unes des questions auxquelles s'attaque Véronique Bergen dans l'analyse qu'elle nous présente de l'ontologie deleuzienne.

La question de l'univocité de l'être est au coeur de la pensée deleuzienne. Elle constitue d'ailleurs le principal motif philosophique de Différence et répétition (Paris, PUF, 1969) considéré par plusieurs comme le Hauptwerk de Deleuze. L'ontologie deleuzienne a suscité de nombreuses réactions. Alain Badiou a par exemple inscrit la pensée deleuzienne dans le prolongement de l'entreprise heideggerienne. Cependant il ne s'agit pas, pour le Deleuze de Badiou, d'opposer le mystère de l'être à l'étant maîtrisable, mais plutôt d'expérimenter l'être dans un unique plan selon les diverses modalités de la synthèse disjonctive (cf. A. Badiou, Deleuze. "La clameur de l'être », 